\title{
EMRE is essential for mitochondrial calcium uniporter activity in a mouse model
}

\author{
Julia C. Liu, ${ }^{1,2,3}$ Nicole C. Syder, ${ }^{1}$ Nima S. Chorashi, ${ }^{1}$ Thomas B. Willingham, ${ }^{4}$ Randi J. Parks, ${ }^{1}$ \\ Junhui Sun, ${ }^{1}$ Maria M. Fergusson, ${ }^{1,2}$ Jie Liu, ${ }^{2,5}$ Kira M. Holmström, ${ }^{2}$ Sara Menazza, ${ }^{1}$ \\ Danielle A. Springer, ${ }^{6}$ Chengyu Liu, ${ }^{7}$ Brian Glancy, ${ }^{4,8}$ Toren Finkel, ${ }^{2,5}$ and Elizabeth Murphy ${ }^{1}$ \\ ${ }^{1}$ Cardiovascular Branch and 2 Center for Molecular Medicine, National Heart, Lung, and Blood Institute, NIH, Bethesda, \\ Maryland, USA. ${ }^{3}$ Postdoctoral Research Associate Program, National Institute of Ceneral Medical Sciences, NIH, Bethesda, \\ Maryland, USA. ${ }^{4}$ Systems Biology Center, National Heart, Lung, and Blood Institute, NIH, Bethesda, Maryland, USA. \\ ${ }^{5}$ Aging Institute, University of Pittsburgh and University of Pittsburgh Medical Center, Pittsburgh, Pennsylvania, USA. \\ ${ }^{6}$ Murine Phenotyping Core and 7 Transgenic Core, National Heart, Lung, and Blood Institute, NIH, Bethesda, Maryland, USA. \\ ${ }^{8}$ National Institute of Arthritis and Musculoskeletal and Skin Diseases, NIH, Bethesda, Maryland, USA.
}

The mitochondrial calcium uniporter is widely accepted as the primary route of rapid calcium entry into mitochondria, where increases in matrix calcium contribute to bioenergetics but also mitochondrial permeability and cell death. Hence, regulation of uniporter activity is critical to mitochondrial homeostasis. The uniporter subunit EMRE is known to be an essential regulator of the channel-forming protein MCU in cell culture, but EMRE's impact on organismal physiology is less understood. Here we characterize a mouse model of EMRE deletion and show that EMRE is indeed required for mitochondrial calcium uniporter function in vivo. $E M R E^{-/-}$mice are born less frequently; however, the mice that are born are viable, healthy, and do not manifest overt metabolic impairment, at rest or with exercise. Finally, to investigate the role of EMRE in disease processes, we examine the effects of EMRE deletion in a muscular dystrophy model associated with mitochondrial calcium overload.

Conflict of interest: The authors have declared that no conflict of interest exists.

Copyright: (c) 2020, American Society for Clinical Investigation.

Submitted: October 7, 2019

Accepted: January 29, 2020

Published: February 4, 2020.

Reference information: JCI Insight. 2020;5(4):e134063.

https://doi.org/10.1172/jci.

insight.134063.

\section{Introduction}

The uptake of cytosolic calcium by energized mitochondria leads to activation of mitochondrial dehydrogenases (1), generating an increase in $\mathrm{NADH}$, as well as activation of the electron transport chain (2) and ATP synthase (3). Thus, mitochondrial calcium uptake plays a critical role in matching ATP production to increased demand (4), as well as enhancing the generation of antioxidants (5). However, when matrix calcium surpasses threshold amounts, the calcium-sensitive mitochondrial permeability transition pore (PTP) is thought to open, leading to necroptosis and cell death (6). Indeed, this is considered a critical cause of injury in the context of ischemia/reperfusion (I/R) (7). Hence, the uptake of mitochondrial calcium must be tightly regulated. This is achieved through the mitochondrial calcium uniporter, a multiprotein complex in the inner mitochondrial membrane (8).

The molecular identification of protein subunits within the mitochondrial calcium uniporter has opened the door to determining the individual contributions of each subunit to the regulation of calcium uptake. Undertaken first in cell culture, these experiments showed that MCU is the channel-forming subunit in the inner mitochondrial membrane $(9,10)$. Since then, a number of studies have examined the in vivo role of MCU in different tissues and mouse models. Although all mouse models have shown that MCU is required for rapid calcium uptake in isolated mitochondria, the physiological and pathological effects of MCU deletion differ depending on whether $M C U$ is deleted globally (11-13) or in specific tissues, chronically (14-17) or in adulthood (16-18). However, it is clear that MCU is required for rapid mitochondrial calcium uptake.

MCU functions as a tetramer (19) in a complex that includes EF-hand proteins MICU1 (20) and MICU2 (21) along with EMRE (essential MCU regulator). EMRE is a single-pass membrane protein that in cultured cells is essential for calcium uptake (22). Studies suggest that EMRE acts as a molecular scaffold, interacting with MCU in its transmembrane domain and with MICU1 in the intermembrane space (2225). EMRE appears to be present only in the metazoan lineage; however, EMRE must be coexpressed with 
human MCU to reconstitute mitochondrial calcium uniporter activity in the yeast Saccharomyces cerevisiae, which lacks MCU (26). To date, it is unknown whether EMRE might impart a further layer of regulation on the uniporter in the organisms in which it is present.

Recently, we generated a mouse model of mitochondrial calcium overload stemming from the deletion of MICU1 (27), which functions in a gatekeeping capacity to inhibit calcium uptake in basal conditions $(28,29)$. Loss of MICU1 led to a perinatal lethality rate of approximately $85 \%$; furthermore, the small fraction of $M I C U 1^{-1-}$ mice that survived to weaning exhibited ataxia and muscle weakness, reminiscent of human patients with loss-of-function mutations in MICU1 (30). Remarkably, with age the MICU1 $1^{-/}$mice seemed to improve in phenotype as well as regain close-to-normal mitochondrial calcium homeostasis, concurrent with a decrease in EMRE protein expression. These data suggest that changes in EMRE expression may be a physiological means to modulate uniporter activity. We therefore first sought to evaluate the effects of EMRE on uniporter regulation in vivo.

Here we characterize a mouse model of EMRE deletion, and show that without EMRE protein, mitochondria are unable to rapidly take up calcium. Although $E M R E^{-/-}$mice are born less frequently, in an outbred background viable mice can be obtained that appear healthy and active, albeit smaller than wildtype $(W T)$ littermate controls. $E M R E^{-/-}$mice have unaltered basal metabolism and are also not significantly impaired in exercise capacity. Although $E M R E^{-/}$mitochondria are resistant to calcium-induced PTP opening, $E M R E^{-/-}$hearts are not protected from I/R injury. Strikingly, EMRE protein expression is upregulated in a mouse model of muscular dystrophy.

\section{Results}

We generated mice with whole-body deletion of EMRE by using CRISPR/Cas9 gene editing targeting the first exon of the EMRE gene, resulting in the deletion of a total of 118 base pairs (Supplemental Figure 1A; supplemental material available online with this article; https://doi.org/10.1172/jci.insight.134063DS1), as previously described (27). We verified by Western blot that in $E M R E^{-/-}$mice, EMRE protein was absent in tissues including heart (Figure 1A), liver, and brain (Supplemental Figure 1B). The uniporter components MCU and MICU1 were still present, albeit slightly reduced, after EMRE deletion. We next assessed the size of the MCU complex with and without EMRE by blue native polyacrylamide gel electrophoresis (BN-PAGE) using heart mitochondria (Figure 1B). In the absence of EMRE, MCU antibody detects a protein complex at approximately $300 \mathrm{kDa}$, which is substantially smaller than the WT complex that runs at about $900 \mathrm{kDa}$, suggesting that loss of EMRE disrupts the stoichiometry of the uniporter complex, as has been reported in cultured cells (22-24).

To determine whether EMRE loss affects the activity of the mitochondrial calcium uniporter, we assessed calcium uptake in mitochondria isolated from $E M R E^{-/-}$mouse tissues and found they were unable to take up calcium (Figure 1C and Supplemental Figure 1, C and D). The absence of mitochondrial uptake is similar to that observed with MCU deletion (Supplemental Figure 1E and ref. 12), and is consistent with evidence in cell culture models that EMRE is essential for calcium uptake $(22,23)$. We further showed in isolated whole flexor digitorum brevis (FDB) muscle fibers that intramitochondrial calcium levels rise significantly higher in $W T$ fibers upon caffeine stimulation than in $E M R E^{-/-}$fibers (Supplemental Figure $1 F)$. Moreover, monitoring the absorbance of mitochondria as a measure of opening of the PTP after the addition of large amounts of extramitochondrial calcium showed that mitochondrial swelling was evident in $W T$ mitochondria but not in $E M R E^{-/-}$mitochondria, demonstrating that EMRE is required for calcium-induced PTP opening (Figure 1D and Supplemental Figure 1G). We isolated primary mouse embryonic fibroblasts (MEFs) from E10 embryos to confirm that $E M R E^{-/-}$cells lack EMRE expression and can no longer take up calcium. After immortalizing these MEFs, we re-expressed a V5-tagged EMRE transgene in $E M R E^{-/-}$MEFs via lentiviral infection. Despite expressing EMRE at lower levels than WT MEFs (Figure $1 \mathrm{E})$, the $E M R E^{-/-}$cells with reconstituted EMRE protein displayed a restored capacity for mitochondrial calcium uptake (Figure 1F). Although uptake rates were somewhat slower (Supplemental Figure 1H), probably due to lower EMRE expression, this rescue demonstrates that EMRE is required for calcium uptake.

We next sought to determine whether EMRE deletion impacts normal mitochondrial function. We evaluated oxygen consumption using a Clark-type electrode, and found no significant difference in the rates of oxygen consumption in mitochondria isolated from $W T$ and $E M R E^{-/-}$heart (Figure 2A) or liver (Supplemental Figure $2 \mathrm{~A}$ ). The respiratory control ratio was likewise similar between $W T$ and $E M R E^{-/-}$in those tissues (Figure $2 \mathrm{~B}$ and Supplemental Figure $2 \mathrm{~B}$ ), suggesting that $E M R E^{-/}$mitochondria have normal baseline respiratory function 
A

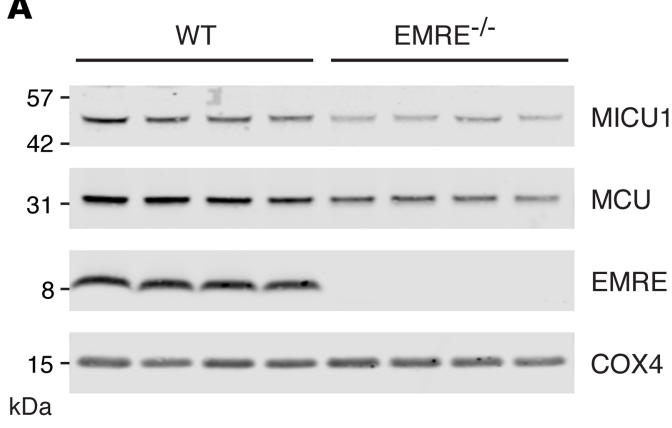

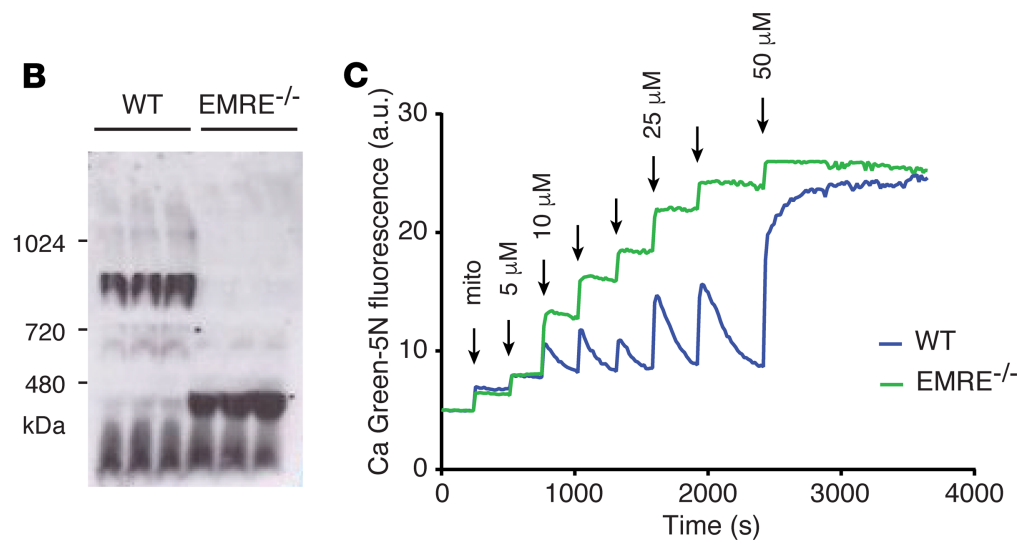

D

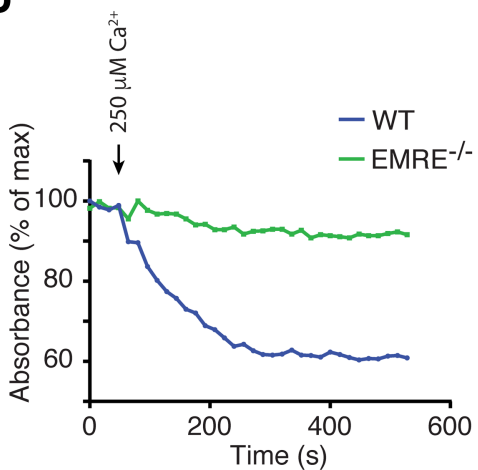

E

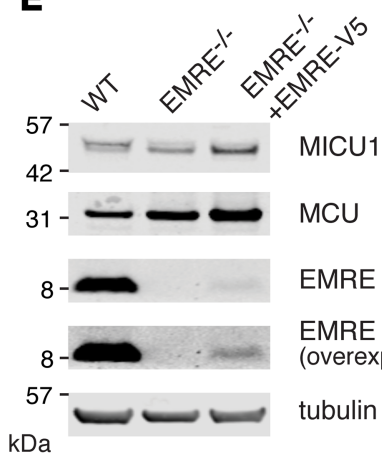

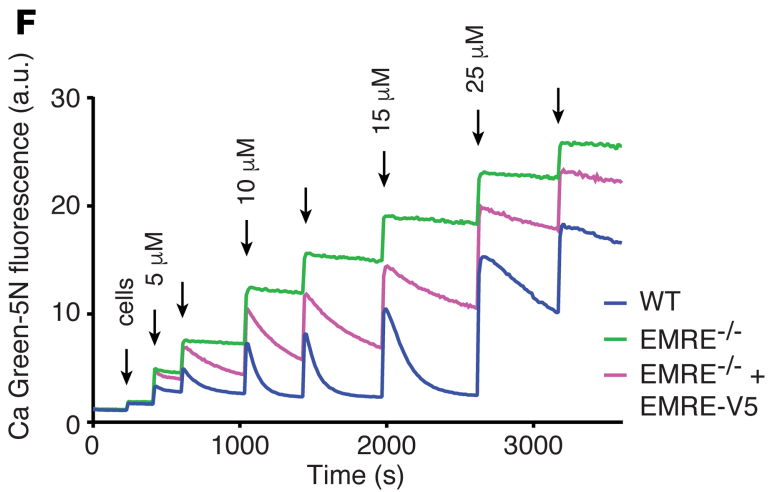

Figure 1. EMRE is required for rapid mitochondrial calcium uptake. (A) Western blot analysis of MICU1, MCU, and EMRE protein expression in isolated mitochondria from WT and $E M R E^{-/-}$hearts ( $n=4$ biological replicates per group). COX4 serves as a mitochondrial loading control. Molecular weights from the protein ladder are indicated on the left. (B) BN-PACE analysis of isolated mitochondria from WT and EMRE ${ }^{-/-}$hearts, immunoblotted with MCU antibody ( $n=3$ technical replicates per group). Data are representative of at least $n=3$ experiments on biological replicates. Molecular weights from the protein ladder are indicated on the left. (C) Extramitochondrial calcium traces with isolated mitochondria from WT and EMRE ${ }^{-/-}$hearts using the calcium indicator dye Calcium Green-5N. Arrows represent calcium addition of the concentration last indicated. Data are representative of at least $n=3$ experiments on biological replicates. (D) Mitochondrial swelling assay measured by absorbance of isolated mitochondria from WT and EMRE-/- hearts in response to a $250 \mu \mathrm{M}$ addition of calcium. Data are representative of at least $n=3$ experiments on biological replicates. (E) Western blot analysis of MICU1, MCU, and EMRE protein expression in MEFs isolated from WT and EMRE ${ }^{-/-}$embryos that were stably transduced with either an empty vector or with a vector encoding a V5 epitope-tagged EMRE construct. Tubulin serves as a loading control. Molecular weights from the protein ladder are indicated on the left. (F) Representative traces of cytosolic calcium measurements in permeabilized MEFs using Calcium Green-5N. Arrows represent calcium addition of the concentration last indicated. Shown are WT MEFs, EMRE-/- MEFs, or EMRE $E^{-/-}$MEFs reconstituted with V5 epitope-tagged EMRE transgene. Data are representative of at least $n=5$ experiments on independent cell cultures.

despite loss of mitochondrial calcium uniporter activity. To further confirm that mitochondrial respiration is unaffected by $E M R E$ deletion, we performed Seahorse analysis on $W T$ and $E M R E^{-/-} M E F$ s to determine their basal and maximal rates of respiration and found no significant difference between cells of these 2 genotypes (Figure 2C). We next sought to determine whether EMRE deletion affects levels of calcium in the matrix by measuring basal mitochondrial calcium. As might be expected for mitochondria unable to take up calcium through the uniporter, $E M R E^{-/}$liver and heart mitochondria had less total and free matrix calcium, respectively, compared with WT mitochondria (Figure 2D and Supplemental Figure 2C).

To determine the importance of EMRE in vivo, we next assessed the phenotypic consequences of loss of EMRE. Although EMRE deletion results in near-total embryonic lethality in inbred C57BL/6N (B6N) mice (Supplemental Figure 3A), $E M R E^{-/-}$mice are viable in a mixed background when crossed to outbred CD-1 mice. Hybrid B6N-CD-1 $E M R E^{-/-}$mice are born at roughly one-fifth the rate expected by Mendelian genetics (Figure 3A); this birth rate increases with another generation of outcrossing but remains less than half of the expected number (Supplemental Figure 3B). Once born, $E M R E^{-/-}$mice appear healthy, active, and almost indistinguishable from $W T$ littermates. However, $E M R E^{-/-}$mice have slightly reduced body weight compared with WT controls (Figure 3B), and females show a slight decrease in fat mass (Supplemental Figure 3C). To determine whether $E M R E^{-/-}$mice exhibited any basal metabolic phenotype, we assessed their total body oxygen consumption and carbon dioxide production in metabolic cages and 


\section{A}

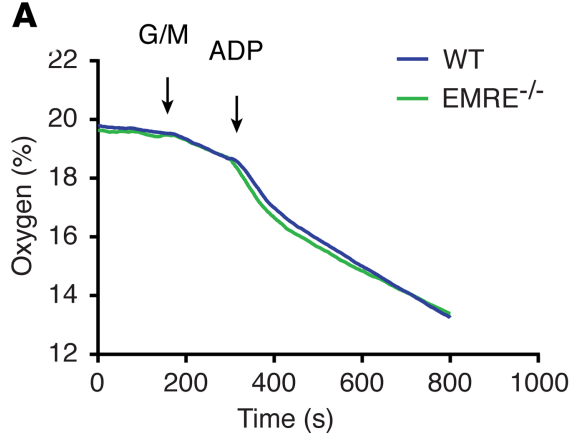

C

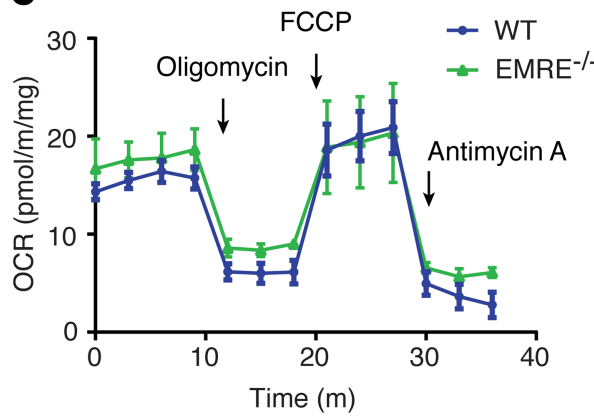

B

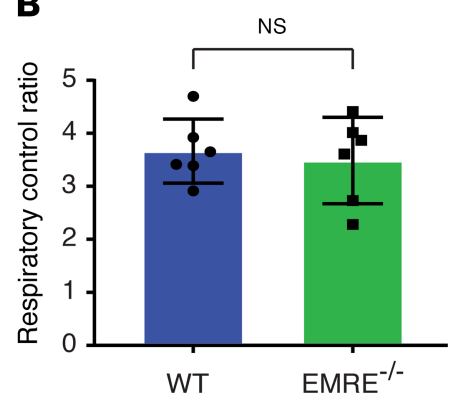

D

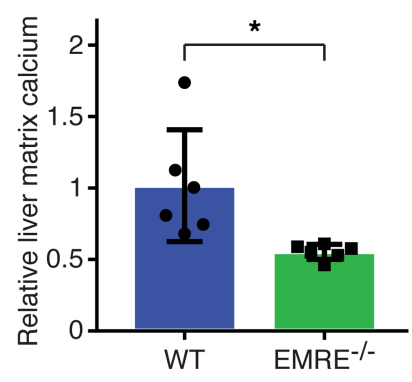

Figure 2. Basal metabolism is not markedly altered with loss of EMRE. (A) Traces of the oxygen consumption observed by Clark-type electrode in isolated WT and $E M R E^{-/-}$heart mitochondria given glutamate and malate (G/M) as substrates, followed by ADP. Data are representative of $n=6$ experiments on biological replicates. (B) Quantification of the respiratory control ratio (RCR; state 3/state 4 respiration) in WT and $E M R E^{-/-}$heart mitochondria ( $n=6$ biological replicates per group). (C) Seahorse X-24 analysis of oxygen consumption rate (OCR) in WT and EMRE ${ }^{-1-}$ MEFs under basal conditions or following the addition of oligomycin, carbonyl cyanide 4-(trifluoromethoxy)phenylhydrazone (FCCP), or antimycin A ( $n=4$ technical replicates per group). Data are representative of $n=3$ experiments on independent cell cultures. (D) Relative levels of matrix calcium in isolated liver mitochondria from WT and EMRE ${ }^{-/-}$mice, measured with a colorimetric calcium assay ( $n=6$ biological replicates per group). Data are represented as mean $\pm S D$; significance was determined by unpaired $t$ test with Welch's correction. NS, not significant; ${ }^{*} P<0.05$.

found no significant difference when compared with $W T$ mice (Figure 3, C and D). Voluntary physical activity was also indistinguishable between $W T$ and $E M R E^{-/-}$mice (Supplemental Figure 3D). Given the importance of mitochondrial calcium in regulating bioenergetics, we next tested whether $E M R E^{-/-}$deletion led to defects in exercise capacity. We measured the ability of $W T$ and $E M R E^{-/-}$mice to sprint uphill on an inclined treadmill at continually increasing speed. In this high intensity protocol, the $E M R E^{-/-}$mice were capable of comparable maximal work relative to $W T$ mice (Figure $3 \mathrm{E}$ ). $E M R E^{-/-}$mice also exhibited similar grip strength as $W T$ mice (Figure $3 \mathrm{~F}$ ). Furthermore, we found no significant difference between $W T$ and $E M R E^{-/-}$mice when we assessed maximal isometric torque generated by in vivo gastrocnemius muscle contraction (Supplemental Figure 3E). These data suggest that loss of EMRE has a substantial effect during development, as a substantial percentage of mice with germline EMRE deletion do not survive. However, mice surviving to adulthood without EMRE do not experience significant impairment of normal behavior and exercise capacity, despite functional loss of mitochondrial calcium uptake. Reasoning that altered mitochondrial calcium efflux might provide a possible compensatory mechanism for the loss of calcium uptake, we measured the protein levels of NCLX as well as the PTP sensitizer cyclophilin D, but did not observe differences between $W T$ and $E M R E^{-/-}$heart or brain lysates (Supplemental Figure 3, F and G).

We further compared basal heart structure and function in $E M R E^{-/-}$and $W T$ mice, reasoning that mitochondrial calcium regulation might be particularly important in cardiac tissue. No overt histological differences were observed between $W T$ and $E M R E^{-/-}$hearts (Supplemental Figure 4A). Furthermore, echocardiography analysis did not reveal any substantial baseline differences between $E M R E^{-/-}$and $W T$ hearts (Figure 4, A and B, and Supplemental Figure 4C). We then assessed whether loss of EMRE affected heart function during increased workload caused by $\beta$-adrenergic stimulation. Echocardiography performed before and after isoproterenol injection revealed similar increases in ejection fraction 
A
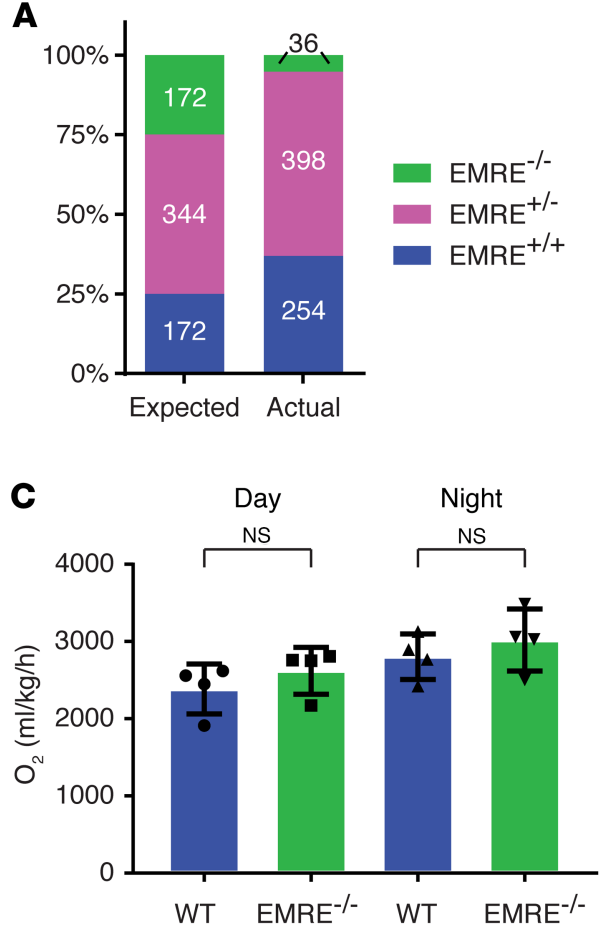

E

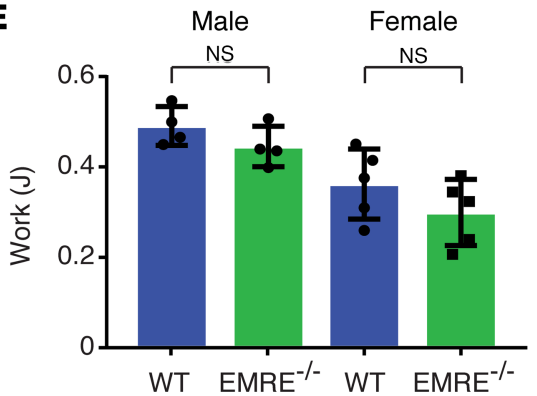

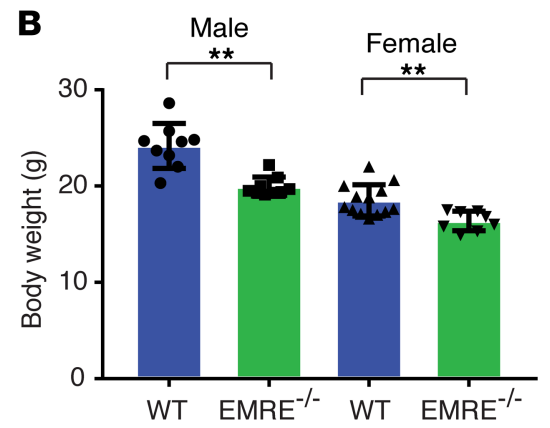

D
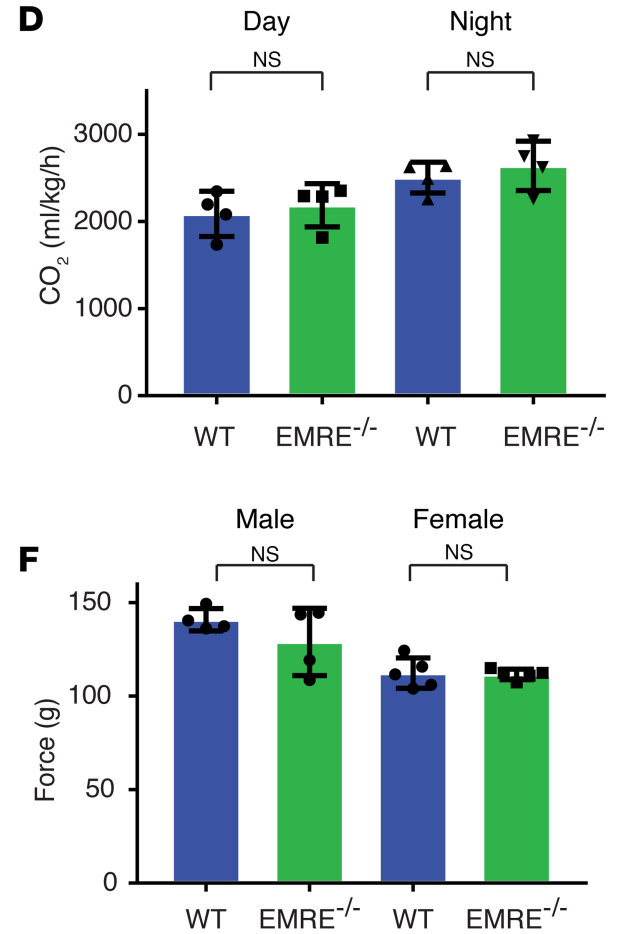

Figure 3. Characterization of EMRE ${ }^{-/-}$mouse phenotype and metabolism. (A) Expected and observed percentage of $E M R E^{+/+}, E M R E^{+/-}$, and $E M R E^{-/-}$mice born to heterozygous F1 (C57B6N $\times$CD-1) parents. The actual number of expected and observed mice is shown within the corresponding bar from a total of 688 offspring. (B) Body weights for 4-week-old $W T$ and $E M R E^{-/-}$male ( $n=9$ biological replicates per group) and female ( $n=13 \mathrm{WT} ; n=8 \mathrm{EMRE}^{-/-}$, all biological replicates) mice. (C) Average total body oxygen consumption in $W T$ and $E M R E^{-/-}$( $n=4$ biological replicates per group) mice during day and night conditions. (D) Average total body carbon dioxide generation in WT and $E M R E^{-/-}$( $n=4$ biological replicates per group) mice during day and night conditions. (E) Assessment of skeletal muscle function in WT and EMRE ${ }^{-/-}$male ( $n$ $=4$ biological replicates per group) and female ( $n=5$ biological replicates per group) mice using maximal work performed on an inclined treadmill test. (F) Grip strength assessment for WT and $E M R E^{-/-}$male ( $n=4$ biological replicates per group) and female ( $n=5$ biological replicates per group) mice. Data are represented as mean \pm SD. WT and EMRE ${ }^{-/-}$mice were compared within each sex or condition using unpaired $t$ test with Welch's correction. NS, not significant; ${ }^{* *} P<0.01$.

and fractional shortening in $W T$ and $E M R E^{-/-}$mice (Figure 4, C and D, and Supplemental Figure 4B), suggesting that chronic EMRE deletion has little effect on cardiac response to this form of acute stress.

Cardiac injury after $\mathrm{I} / \mathrm{R}$ is thought to be associated with calcium entry into mitochondria, which contributes to PTP opening and cell death $(31,32)$. Therefore, loss of EMRE protein and hence loss of mitochondrial calcium uptake ability would be predicted to protect the heart against I/R injury. Instead, hearts from $E M R E^{-/-}$mice subjected to an ex vivo Langendorff perfusion model of global I/R showed no improvement relative to $W T$ hearts in terms of recovery of left ventricular pressure or infarct size (Figure 4, E and F, and Supplemental Figure 4F). Treatment with cyclosporine A (CsA) to inhibit PTP opening conferred protection to $W T$ hearts, as expected; however, CsA did not have an effect on $E M R E^{-/-}$hearts (Figure 4, E and F, and Supplemental Figure $4 \mathrm{~F}$ ). We also examined whether $E M R E^{+/-}$ 
A

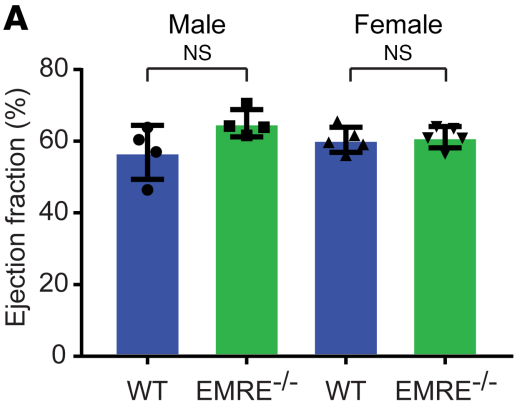

C

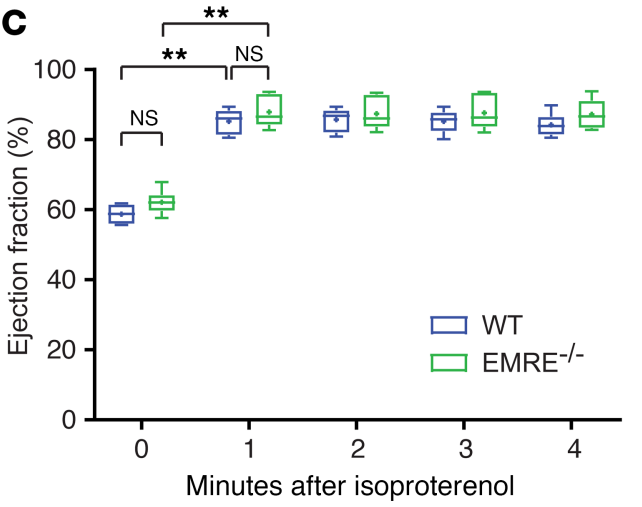

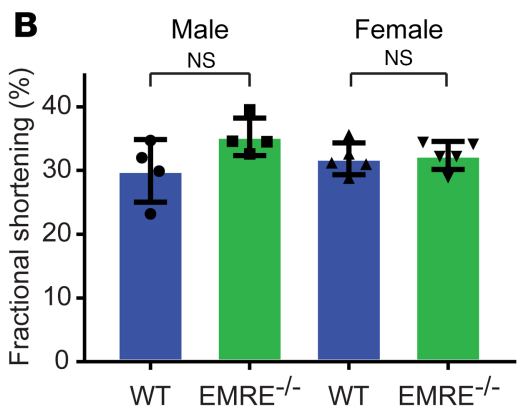

D

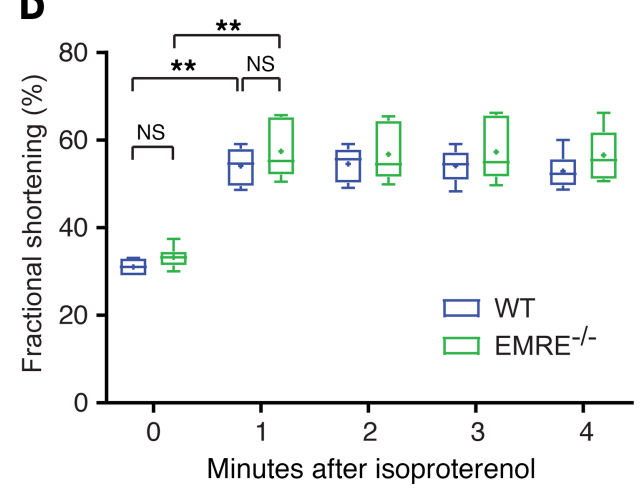

\section{E}

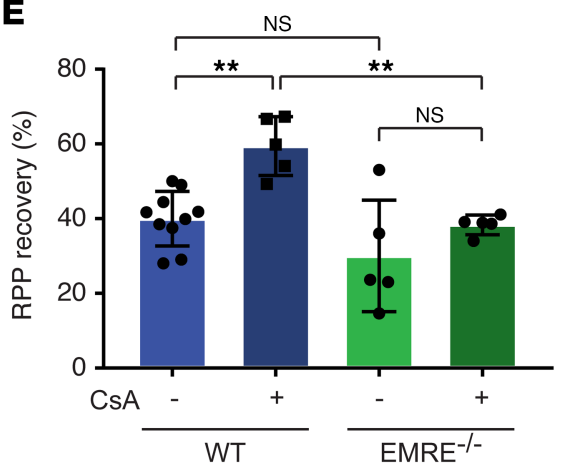

$\mathbf{F}$

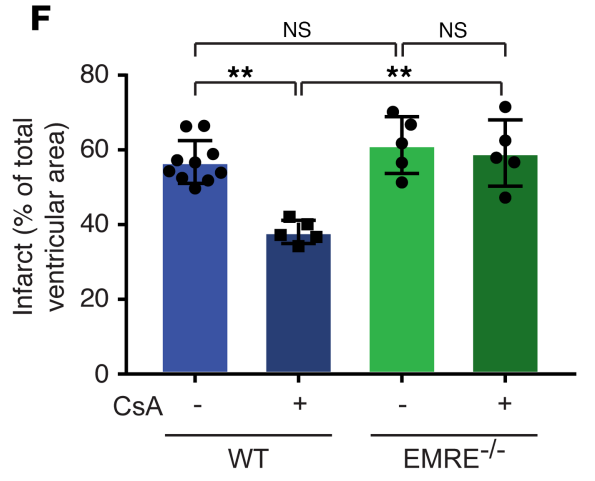

Figure 4. Deletion of EMRE does not alter cardiac function or cardiac injury after ischemia/reperfusion. (A and B) Echocardiography analysis of WT and $E M R E^{-/-}$male ( $n=4$ biological replicates per group) and female ( $n=5$ biological replicates per group) mice assessing ejection fraction (A) and fractional shortening (B). Data are represented as mean \pm SD. WT and $E M R E^{-/-}$mice were compared within each sex using unpaired $t$ test with Welch's correction. NS, not significant. (C and D) Echocardiography analysis at baseline and 1, 2, 3, and 4 minutes following isoproterenol (2 $\mathrm{mg} / \mathrm{kg})$ injection of male $W T$ and $E M R E^{---}(n=6$ biological replicates per group) mice assessing ejection fraction (C) and fractional shortening (D). Boxes were plotted extending from the 25th to 75th percentiles, with the line in between showing the median; whiskers extend from the minimum to the maximum. The "+" sign indicates the mean. Data were first compared by 2-way ANOVA followed by Bonferroni's multiple-comparisons test to determine significance for each pair of data sets. ${ }^{* *} P<0.01$; NS, not significant. (E and $\mathbf{F}$ ) Assessment in the hearts of $W T$ and $E M R E^{-/-}$mice with and without cyclosporine A (CsA, 0.2 $\mu \mathrm{M})(n=10$ biological replicates for WT without CsA, $n=5$ biological replicates for remaining groups) for 5 minutes before ischemia, following 20 minutes of global ischemia and 90 minutes of reperfusion of the rate pressure product (RPP; heart rate times systolic blood pressure) (E) and infarct size relative to the area at risk, which is the entire left ventricle (F). Data are represented as mean \pm SD. Data were first compared by 1-way ANOVA followed by unpaired $t$ test with Welch's correction to determine significance for each pair of data sets. ${ }^{* *} P<0.01$; NS, not significant.

hearts would be protected from I/R injury. We previously showed that EMRE protein expression in $E M R E^{+/-}$mice are unchanged from $W T$ levels (27). We postulated that perhaps newly translated EMRE protein affects the quantity or stoichiometry of MCU-EMRE complexes during I/R and might contribute to injury, in which case $E M R E^{+/-}$mice might be protected. However, $E M R E^{+/-}$and $W T$ hearts were similarly affected in the Langendorff I/R model (Supplemental Figure 4, D-F). 
A

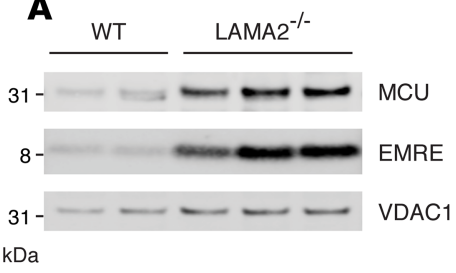

E

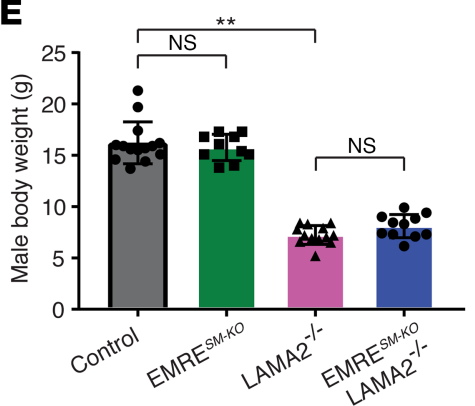

H

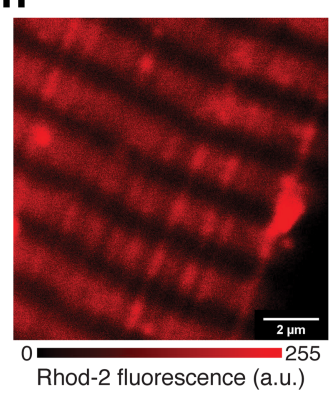

B

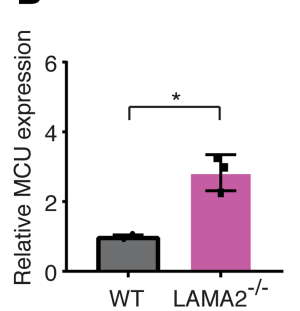

$\mathbf{F}$
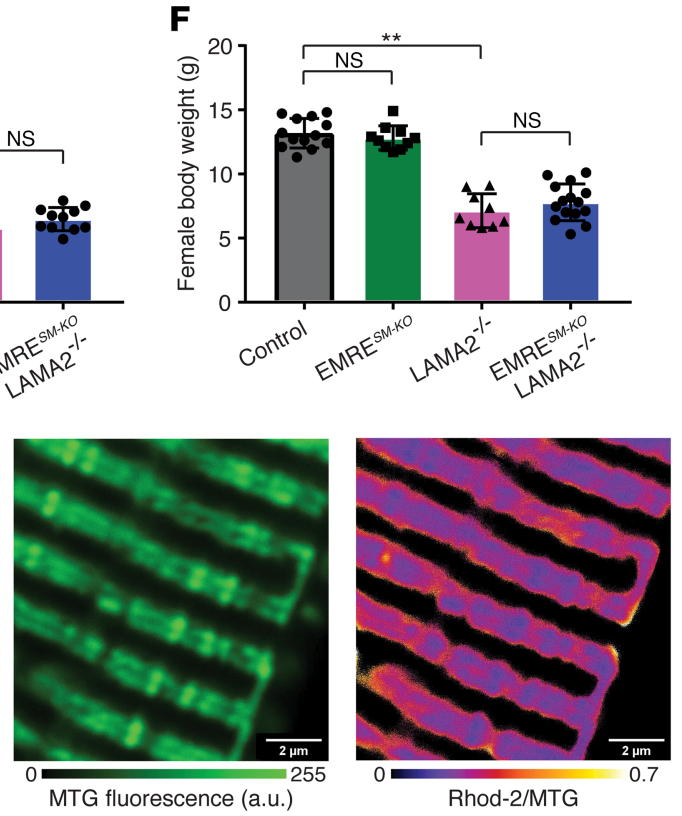

C

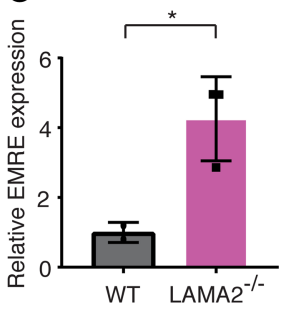

D
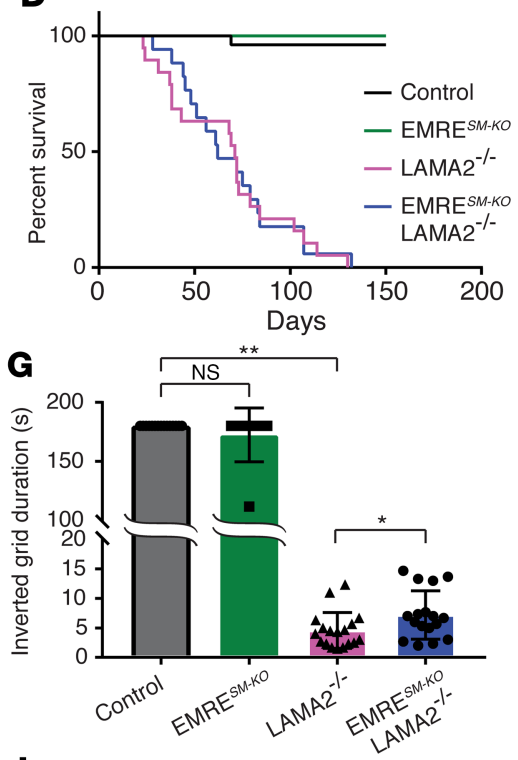

I

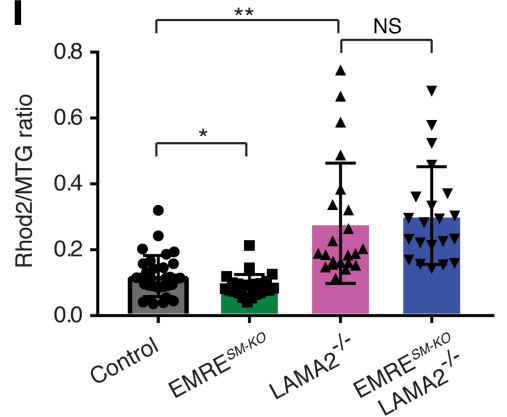

Figure 5. Characterization of the role of EMRE in a muscular dystrophy mouse model exhibiting mitochondrial calcium overload. (A) Western blot analysis of MCU and EMRE protein expression in quadriceps muscle from WT ( $n=2$ biological replicates) and LAMA2 $2^{-1-}(n=3$ biological replicates) mice. VDAC1 serves as a mitochondrial loading control. Molecular weights from the protein ladder are indicated on the left. (B and C) Quantification of MCU (B) and EMRE (C) protein expression in A, normalized to VDAC1. Data are represented as mean \pm SD; results of unpaired $t$ test with Welch's correction. ${ }^{*} P<0.05$. (D) Survival curve for control $(n=26), \operatorname{EMRE}^{S M-K O}(n=16), \operatorname{LAMA2}^{-1-}(n=19)$, and $E M R E^{S M-K O} \operatorname{LAMA2}^{-1-}(n=17)$ mice (all biological replicates). (E) Body weights for 4-week-old male control $(n=14), \operatorname{EMRE}^{S M-K O}(n=10), \operatorname{LAMA2}^{-1-}(n=13)$, and $E M R E^{S M-K O} \operatorname{LAMA2}^{-1-}(n=11)$ mice (all biological replicates). (F) Body weights for 4-week-old female control $(n=13), \operatorname{EMRE}^{S M-K O}(n=10), \operatorname{LAMA2}^{-1-}(n=9)$, and $\operatorname{EMRE}^{S M-K O} \operatorname{LAMA2}^{-1-}(n=15)$ mice (all biological replicates). (C) Assessment of muscle strength using an inverted grid test on 4-week-old control $(n=15), \operatorname{EMRE}^{S M-K O}(n=9), \operatorname{LAMA2}^{-1-}(n=18)$, and $E M R E^{S M-K O} L_{A M A 2^{-1-}}(n=17)$ mice $(a l l$ biological replicates). (H) Representative image of an isolated flexor digitorum brevis (FDB) muscle fiber loaded with the fluorescent calcium-sensitive dye Rhod-2 AM (in red), the mitochondria mass stain MitoTracker Green (in green), and the ratio of Rhod-2 AM to MitoTracker Green (heatmap). (I) Relative levels of matrix calcium in FDBs isolated from control $\left(n=32\right.$ cells from $n=5$ mice), $E M R E^{S M-K O}\left(n=25\right.$ cells from $n=4$ mice), LAMA2 $2^{-1-}$ ( $n=22$ cells from $n$ $=3$ mice), and $E M R E^{S M-K O} L A M A 2^{-1-}(n=21$ cells from $n=3$ mice) mice (biological replicates) as measured by the ratio of Rhod-2 AM to MitoTracker Green. Data are represented as mean $\pm \mathrm{SD}$. Data were first compared by 1-way ANOVA followed by unpaired $t$ test with Welch's correction to determine significance for each pair of data sets. ${ }^{*} P<0.05 ;{ }^{* *} P<0.01$.

Mitochondrial calcium overload is associated with a number of diseases, including muscular dystrophies (33), heart failure (34), and neurodegenerative disorders (35). Because $M I C U 1^{-{ }^{-}}$mice, which exhibit mitochondrial calcium overload and a profound skeletal myopathy, show age-dependent downregulation of EMRE associated with improved mitochondrial calcium handling (27), we hypothesized that EMRE might also be reduced in mouse models of muscular dystrophy. We used a mouse model of congenital merosin-deficient muscular dystrophy (36), in which the LAMA2 gene encoding the $\alpha 2$ subunit of laminin, an extracellular protein integral to the basement membrane, is deleted. Surprisingly, by Western blot we observed higher MCU levels and even more dramatically elevated EMRE expression in skeletal muscle from $L A M A 2^{-1-}$ mice (Figure 5, A-C). Consistently, in the $m d x$ mouse model of Duchenne muscular dystrophy, expression of MCU and EMRE protein also showed a trend to be increased (Supplemental Figure 5A). To determine whether this increase in EMRE contributes to the disease, we deleted EMRE in $L A M A 2^{-1-}$ skeletal muscle. We crossed the LAMA2 muscular dystrophy mouse model to C57BL/6 mice with skeletal 
muscle deletion of $E M R E$ (herein called $E M R E^{S M-K O}$ ), which we generated by crossing $E M R E^{\text {Affl }}$ conditional mice (37) to transgenic MCK-Cre mice (Supplemental Figure 5B and ref. 38). This strategy allowed comparison to the expression of human laminin $\alpha 2$ under the MCK promoter, which was shown to rescue the dystrophic phenotype in these $L A M A 2^{-/-}$mice in terms of lifespan, size, and appearance (36), and additionally avoided any phenotypic improvement merely due to introducing the mixed background of germline $E M R E^{-/-}$mice. We expected that blocking uniporter activity via $E M R E$ deletion would reduce mitochondrial calcium overload and subsequent PTP opening; indeed, deletion of the PTP sensitizer cyclophilin D was previously shown to improve the survival and muscle integrity of $L A M A 2^{-1-}$ mice (39). However, we observed no difference in lifespan between $L A M A 2^{-/-}$and $E M R E^{S M-K O} L A M A 2^{-/-}$mice (Figure 5D). Furthermore, although they were both significantly smaller than control mice, $L A M A 2^{-/-}$and $E M R E^{S M-K O} L A M A 2^{-1-}$ mice had similar body weights (Figure 5, E and F, and Supplemental Figure 5C). Phenotypic assessment of skeletal muscle strength in an inverted grid test revealed significant impairment in $L A M A 2^{-1-}$ mice, with a statistically significant, but very modest, improvement in $E M R E^{S M-K O} L A M A 2^{-1-}$ mice (Figure 5G).

We next sought to understand why EMRE deletion in the skeletal muscle conferred little or no functional benefit to the $L A M A 2^{-/-}$mice by first confirming the premise that this muscular dystrophy model suffers from mitochondrial calcium overload. We therefore measured mitochondrial calcium levels in individual isolated FDB muscle fibers via the calcium-sensitive, mitochondria-localized dye Rhod-2 normalized to the mitochondrial mass marker MitoTracker Green (Figure $5 \mathrm{H}$ ). We found a slight but significant decrease in mitochondrial calcium in the $E M R E^{S M-K O}$ fibers compared with the control fibers (Figure 5I). As expected, $L A M A 2^{-/-}$fibers exhibited higher mitochondrial calcium levels than control fibers. Surprisingly, EMRE $E^{S M-K O}$ $L A M A 2^{-/-}$fibers had elevated calcium levels indistinguishable from $L A M A 2^{-/-}$fibers, suggesting a role for slow mitochondrial calcium uptake pathways.

\section{Discussion}

Although previous studies have shown that loss of EMRE blocks mitochondrial calcium uptake, this is the first study to our knowledge to characterize the physiological effects of in vivo loss of EMRE in a mouse model. Despite its relatively recent appearance in evolution, EMRE has proven to be essential for calcium uptake by the mitochondrial calcium uniporter in cell culture (22), recently in Drosophila melanogaster (40), and now in this study in a vertebrate. Although MCU is still present in mitochondria despite EMRE deletion, our BN-PAGE data show that the MCU complex is much smaller, consistent with BN-PAGE results from cultured cells (22). As EMRE has been suggested to function as a scaffold for different components of the uniporter (23), in its absence it is possible that subunits such as those in the MICU family are unable to form a stable complex with MCU. Disruption of the binding of uniporter complex members to one another may reduce protein stability and contribute to the reduced overall expression of MCU and MICU1 observed with EMRE deletion. Moreover, EMRE is potentially important for the dimerization of 2 tetrameric MCU complexes with each other (19). Thus, the importance of EMRE in the assembly of the uniporter likely underlies its essential role in mediating calcium uptake in multiple metazoan organisms.

The necessity of breeding mice in a mixed genetic background to obtain viable $E M R E^{-/-}$mice is reminiscent of germline $M C U^{-1-}$ mice, and indeed both genotypes are born at similarly low rates. This circumstance in which a genetic mutation manifests less severely in an outbred strain is fairly common (41-44). The obstacle that EMRE or MCU deletion poses to completing embryonic development might be attributed to the recently discovered role of mitochondrial calcium uptake in regulating cell cycle progression $(45,46)$, and the outbred background might better tolerate the loss of uniporter activity in the developing embryo. One limitation of the study is that EMRE was deleted in the germline, and we cannot exclude the possibility that compensatory pathways were activated. Furthermore, the $E M R E^{-/-}$mice are not born at the expected ratio, and therefore the mice that are born may be selected for those which have adaptations.

The phenotype of $E M R E^{-/-}$mice was reminiscent of that of $M C U^{-1-}$ mice (12). Germline deletion of either EMRE or MCU resulted in mitochondria that did not take up added calcium and did not undergo PTP opening on addition of calcium. Despite the lack of calcium-induced PTP opening, neither genotype was protected from I/R injury. Basal metabolism measured in vivo as well as in mitochondria and cells was not affected in $E M R E^{-/-}$or $M C U^{-1-}$ mice relative to $W T$ mice. This similarity is probably not surprising, as loss of EMRE blocks mitochondrial calcium uptake via MCU. Deletion of EMRE and MCU had more subtle effects on energetic capacity and exercise than expected; however, $M C U^{-/-}$mice did exhibit minor impairment relative to $W T$ in skeletal muscle performance (12), while a similar trend 
in $E M R E^{-/-}$mice did not reach significance. The surprisingly mild phenotype resulting from deletion of EMRE or MCU underscores the need to more thoroughly investigate mitochondrial adaptation to the loss of uniporter function. One might conjecture that as-yet unknown compensatory mechanisms may allow $M C U^{-/-}$or $E M R E^{-/-}$mitochondria to decrease reliance on calcium as a signal to stimulate energy production. Another perhaps more likely possibility is that mitochondria lacking a functional calcium uniporter upregulate alternative, uniporter-independent pathways to import calcium into the matrix. This hypothesis is supported by evidence that basal calcium levels in the mitochondrial matrix do not always decrease in the absence of uniporter activity. For instance, some previous studies on various $M C U$-null mouse models reported lower matrix calcium $(11,12)$, whereas others have reported calcium levels similar to those of controls $(16,18)$. Interestingly, NCLX protein expression did not change in $E M R E^{-/-}$mitochondria, indicating that in this context downregulating calcium efflux does not significantly contribute to maintaining matrix calcium levels.

Adaptation to loss of uniporter function via deletion of $M C U$ or $E M R E$ is also likely to be context dependent, as illustrated by discrepancies in I/R injury results obtained from different models of $M C U$ deletion or inhibition. The infarct size is reduced in hearts from mice in which $M C U$ is deleted in adulthood via a tamoxifen-inducible heart-specific Cre $(16,18)$, showing that acute gene deletion supports the predicted role of the mitochondrial calcium uptake in contributing to I/R injury. In contrast, $M C U$ deletion or expression of dominant-negative $M C U$ in the germline did not affect infarct size or recovery after I/R $(12,14)$, and here we find similar results with germline deletion of EMRE. These observations suggest that chronic loss of uniporter activity leads to mitochondrial reconfiguration and adaptation. Recently, analysis of the phosphoproteome of $M C U^{-1-}$ heart mitochondria identified changes including an increase in the phosphorylation of cyclophilin $\mathrm{D}$, linked to a lower mitochondrial calcium retention capacity (13). Hence, despite lacking rapid calcium uptake through the uniporter, $M C U^{-/}$or $E M R E^{-/-}$ mitochondria may be rewired in ways that render them still susceptible to PTP opening through a pathway not inhibitable by CsA. Determining the features of this rewiring, its mechanism, and how it develops over time is critical before we can effectively target the uniporter in diseases and disorders characterized by mitochondrial calcium overload.

We noted that the muscle-specific deletion of EMRE in the LAMA2 muscular dystrophy mouse model was unable to rescue shortened lifespan and severe muscle weakness, likely because basal matrix calcium levels were not significantly lowered in $E M R E^{S M-K O} L A M A 2^{-/-}$muscle fibers when compared with $L A M A 2^{-1-}$ fibers. We did, however, observe some residual EMRE expression in $E M R E^{S M-K O} L A M A 2^{-/-}$muscle (Supplemental Figure 5D) and therefore cannot completely exclude the possibility that MCK-Cre was incompletely efficient at deleting EMRE in these muscle fibers. This residual EMRE expression might also reflect increased infiltration in the disease context of other cell types unaffected by MCK-Cre, such as fibroblasts and immune cells. It is also possible that EMRE and MCU upregulation in $L A M A 2^{-/-}$mice represents an adaptive response to energetic stress due to constant damage and repair in dystrophic muscle. In this scenario, elevating uniporter activity might help mitochondria to keep up with energetic demand. Hence, deletion of EMRE does not substantially improve acute muscle performance because rapid mitochondrial calcium uptake might be necessary to satisfy the bioenergetic requirements of exercise such as the inverted grid. The observed increase in EMRE expression in $L A M A 2^{-1-}$ mice is opposite to the EMRE downregulation seen following MICU1 deletion, although both conditions are associated with elevated mitochondrial calcium levels. It should be noted, however, that muscular dystrophies are also characterized by elevated cytosolic calcium (47). Hence, diseases characterized by mitochondrial calcium overload are not necessarily equivalent. Nonetheless, EMRE protein levels seem to be quite dynamic and appear to respond to a wide variety of mitochondrial and cellular stressors, suggesting EMRE may have evolved to actively regulate uniporter activity to allow tissues to adapt to changing conditions.

\section{Methods}

Mice. The EMRE-knockout mice were originally generated on the C57BL/6N background as described previously (27), but were crossed to CD-1 mice (Charles River) for several generations before they were used in the current study. The EMRE conditional knockout mice were recovered from frozen sperm (strain C57BL/6N-A $\mathrm{A}^{\mathrm{tm} 1 \mathrm{Brd} / \mathrm{a}}$ Smdt1 $1^{\mathrm{tm} \text { la(EUCOMM)Wtsi } / B c m M m u c d)}$ by the Mutant Mouse Resource and Research Center (MMRRC). They were first crossed to ROSA26-FLPo mice (48) (JAX, stock 012930) to excise the selection marker and lacZ reporter gene. Subsequently, these $E M R E^{\text {flfl }}$ mice were crossed with MCK-Cre mice (JAX, stock 006475) 
obtained from Lee Weinstein (National Institute of Diabetes and Digestive and Kidney Diseases, Bethesda, MD). LAMA2 $d y^{W}$ null mutant mice were purchased from JAX (stock 013786). C57BL/10J (JAX, stock 000476) controls and $m d x$ mice (JAX, stock 001801) were gifts from Helene Rosenberg (National Institute of Allergy and Infectious Diseases, Bethesda, MD). $M C U^{-1-}$ mice (12) were maintained in our colony. All animal studies were done in accordance with the NIH Guide for the Care and Use of Laboratory Animals (National Academies Press, 2011) and with the approval of the NHLBI Animal Care and Use Committee.

Cell culture. MEFs were generated from mouse embryos (C57BL/6N-CD1 hybrid background) at approximately E10-E12 using standard methods. MEFs were cultured in growth medium consisting of Dulbecco's modified Eagle's medium (DMEM) supplemented with 10\% fetal bovine serum (FBS), 50 U/ $\mathrm{mL}$ penicillin and $50 \mu \mathrm{g} / \mathrm{mL}$ streptomycin. For reconstitution experiments, $W T$ and $E M R E^{-/-} \mathrm{MEFs}$ were immortalized by serial passaging. The EMRE-V5 vector was cloned into the pLVX lentiviral backbone, and transfected into 293T cells for lentivirus generation using Lipofectamine (manufacturer's protocol). MEFs were infected with lentivirus in the presence of $8 \mu \mathrm{g} / \mathrm{mL}$ polybrene (Millipore) for 24 hours, then selected with $3 \mu \mathrm{g} / \mathrm{mL}$ puromycin.

Mitochondrial isolation. Mitochondria were isolated by standard differential centrifugation procedures, as previously described $(12,27)$. Tissues were first minced in isolation buffer $(225 \mathrm{mM}$ mannitol, 75 $\mathrm{mM}$ sucrose, $5 \mathrm{mM}$ MOPS, $0.5 \mathrm{mM}$ EGTA, $2 \mathrm{mM}$ taurine, $\mathrm{pH}$ adjusted to 7.25) and then homogenized using a Glas-Col homogenizer for 3 to 5 strokes (liver and brain) or an Ultra-Turrax homogenizer (IKA Labortecknik). For heart, trypsin was added to the homogenate for 5 minutes on ice, followed by addition of $0.2 \%$ BSA to stop digestion. For liver and brain, $0.2 \%$ BSA was present in isolation buffer continuously. Homogenates were initially centrifuged at $500 \mathrm{~g}$ and the resulting supernatant was next spun at $11,000 \mathrm{~g}$ to pellet the mitochondria. The final mitochondrial pellet was resuspended in isolation buffer with $0.2 \% \mathrm{BSA}$. Protein content was measured by BCA protein assay (Pierce).

Calcium uptake, swelling, and calcium content. As previously described (27), isolated mitochondria or MEFs were resuspended in a buffer containing $125 \mathrm{mM} \mathrm{KCl}, 2 \mathrm{mM} \mathrm{K} \mathrm{HPO}_{4}, 10 \mu \mathrm{M} \mathrm{EGTA}, 1 \mathrm{mM}$ $\mathrm{MgCl}_{2}, 20 \mathrm{mM}$ HEPES at $\mathrm{pH}$ 7.2, $5 \mathrm{mM}$ glutamate, and $5 \mathrm{mM}$ malate. For MEFs, 0.004\% digitonin was added for cell permeabilization. Calcium uptake was measured with the fluorescent calcium indicator Calcium Green-5N (Thermo Fisher Scientific) at a final concentration of $1 \mu \mathrm{M}$. For quantifying uptake rates, slopes were calculated from linear fits of Calcium Green-5N traces during the first 2 postpeak minutes using GraphPad Prism software. Calcium-induced mitochondrial swelling was measured in isolated mitochondria as a decrease in absorbance at $540 \mathrm{~nm}$ using a microplate reader (FLUOstar Omega, BMG Labtech).

Measurement of intramitochondrial calcium content was performed as previously described (12, 27). Briefly, liver and heart mitochondria were isolated continuously in the presence of $2 \mu \mathrm{M}$ Ru360 (Calbiochem) and $10 \mu \mathrm{M}$ CGP-37157 (Tocris) to inhibit mitochondrial exchange of calcium during the isolation. For total matrix calcium in liver mitochondria, mitochondria were washed in isolation buffer without EGTA, and then pelleted and diluted in $0.6 \mathrm{~N} \mathrm{HCl}$, homogenized, and sonicated. After heating for 30 minutes at $95^{\circ} \mathrm{C}$ and centrifuging for 5 minutes at $10,000 \mathrm{~g}$, supernatants were brought to neutral $\mathrm{pH}$. Calcium concentration was determined spectrophotometrically using the $O$-Cresolphthalein Complexone calcium assay kit (Cayman Chemical). For free matrix calcium in heart mitochondria, mitochondria were loaded with $20 \mu \mathrm{M}$ Fluo-4 AM (Thermo Fisher Scientific) for 30 minutes at room temperature and then washed twice with mitochondrial isolation buffer, and then twice more with buffer without EGTA. Finally, mitochondria were resuspended in buffer containing $137 \mathrm{mM} \mathrm{KCl}, 20 \mathrm{mM} \mathrm{HEPES}$, and $2 \mathrm{mM} \mathrm{K}_{2} \mathrm{HPO}_{4}$ at $\mathrm{pH} 7.15$, and baseline fluorescence was measured on a microplate reader (FLUOstar Omega, BMG Labtech). The $\mathrm{F}_{\max }$ value was obtained by addition of $5 \mu \mathrm{M}$ ionomycin (Tocris) and $10 \mu \mathrm{M}$ calcium, followed by the $\mathrm{F}_{\min }$ value by addition of $1 \mathrm{mM}$ EGTA.

Metabolic studies. Oxygen consumption of isolated mitochondria was measured in a chamber connected to a Clark-type $\mathrm{O}_{2}$ electrode (Instech). Mitochondria were incubated in respiration buffer containing $120 \mathrm{mM}$ $\mathrm{KCl}, 10 \mathrm{mM}$ MOPS, $5 \mathrm{mM} \mathrm{K}_{2} \mathrm{HPO}_{4}, 20 \mu \mathrm{M} \mathrm{EGTA}, 5 \mathrm{mM} \mathrm{MgCl}_{2}$, and $0.2 \% \mathrm{BSA}(\mathrm{pH}$ 7.2). After addition of $10 \mathrm{mM}$ glutamate $/ 5 \mathrm{mM}$ malate, state 3 respiration was measured by addition of $100 \mu \mathrm{M}$ ADP. The respiratory control ratio was determined by the state 3 /state 4 respiration rates. Measurement of intact cellular respiration was performed using the Seahorse XF24 analyzer. Using $25 \mathrm{mM}$ glucose as the extracellular substrate, respiration was measured under the basal condition, in the presence of oligomycin $(0.375 \mu \mathrm{M})$, followed by FCCP $(0.625 \mu \mathrm{M})$, and lastly antimycin A $(1 \mu \mathrm{M})$. Following the experiment, protein content of the cells in each well was determined by BCA assay, and respiration rates were normalized to protein mass. 
Protein extraction from tissues and cells. Quadriceps muscle was dissected out and snap-frozen in liquid nitrogen. While still frozen, samples were ground with a mortar and pestle, then homogenized in ice-cold RIPA buffer (Pierce) with protease and phosphatase inhibitors (Roche) using a Precellys 24 Tissue Homogenizer with the Cryolys cooling option (Bertin Instruments). For MEFs, cell pellets were lysed in Cell Lysis Buffer (Cell Signaling) with protease and phosphatase inhibitors (Roche). After lysis on ice for 30 minutes and centrifugation at $4^{\circ} \mathrm{C}$ at $14,000 \mathrm{~g}$ for 30 minutes, supernatants were collected and protein concentration was measured by BCA protein assay (Pierce).

$B N-P A G E$. Freshly isolated mitochondria were incubated on ice with NativePAGE sample buffer (Invitrogen) and digitonin at a final concentration of 2\%. After centrifugation, NativePAGE 5\% G-250 Sample Additive (Invitrogen) was added to the supernatants to a final concentration of $0.25 \%$. Gel electrophoresis running buffers were prepared according to the manufacturer's protocol for the NativePAGE Novex Bis-Tris Gel System, and electrophoresis was performed at $4^{\circ} \mathrm{C}$. After electrophoresis was complete, gels were transferred to nitrocellulose membranes overnight at $4^{\circ} \mathrm{C}$. Thereafter, membranes were blocked and immunoblotted with primary antibody as described below.

Western blot. Samples were diluted in NuPAGE LDS sample buffer (Invitrogen) and run in MES buffer on NuPAGE Bis-Tris $4 \%$ to $12 \%$ gels (Invitrogen). Gels were transferred to nitrocellulose membranes, which were then blocked and immunoblotted with primary antibodies against EMRE (Santa Cruz Biotechnology, sc-86337), MICU1 (Sigma-Aldrich, HPA037480), MCU (Sigma-Aldrich, HPA016480), NCLX (Santa Cruz Biotechnology, sc-161921), cyclophilin D (Abcam, ab110324), tubulin (Developmental Studies Hybridoma Bank, E7), COX4 (Invitrogen, 20E8C12), and VDAC1 (Santa Cruz Biotechnology, sc-390996). Proteins were visualized using LI-COR IRDye secondary antibodies and an Odyssey CLx imaging system.

Phenotyping and exercise testing. Total body oxygen consumption and carbon dioxide production were measured by an open circuit indirect calorimeter (CLAMS, Columbus Instruments). Mice 5 to 6 months of age were given ad libitum access to food and water and were singly housed 1 day before the experiment in sealed plastic metabolic cages that were connected to $\mathrm{O}_{2}$ and $\mathrm{CO}_{2}$ sensors. After the 1-day acclimation period, energy expenditure and respiratory exchange ration were measured for 3 consecutive days at 30-minute intervals. Over the same time period, voluntary physical activity was measured via counts of ambulation (consecutive beam breaks along the $x$ axis of the cage).

Mice 3 to 4 months of age were tested for exercise capacity with a high intensity, inclined ramp protocol, as previously described (12). Mice were run on a rodent treadmill (Columbus Instruments) at an incline of 20 degrees and at a starting speed of $14 \mathrm{~m} / \mathrm{min}$. Every 5 seconds, the speed was increased by $2 \mathrm{~m} / \mathrm{min}$. Mice ran until failure, which was the last speed in which the mice were capable of staying on the inclined treadmill for the full 5 seconds. Following acclimation and training to run on the treadmill, mice were assessed in 3 trials, each separated by 24 hours. Maximal work was calculated for each of the 3 trials using the formula: work $=$ body weight $(\mathrm{kg}) \times$ vertical distance $(\sin 20 \times$ distance $[\mathrm{m}])$.

Before grip strength testing, mice 3 to 4 months of age were acclimated to the testing room for 30 minutes. Grip strength was measured using a Grip Strength Meter (Columbus Instruments), as previously described (12). Briefly, the grip strength meter was positioned horizontally as the experimenter gently lowered the mice toward the apparatus. Mice were allowed to grasp the smooth, metal, triangular pull bar and were then pulled backward in the horizontal plane. The force applied to the bar at the moment the grasp was released was recorded as the peak tension $(\mathrm{kg})$. The test was repeated 5 consecutive times within the same session.

Muscle strength was assessed in 4-week-old mice with a noninvasive inverted grid suspension test, as previously described (27). Mice were placed on a grid that was slowly turned over $180^{\circ}$, suspending the mice above a padded surface. Latency to fall was timed, with the maximum for any trial set at 180 seconds. The average of 3 trials with rest periods in between was reported per mouse.

In vivo gastrocnemius muscle contractility was measured using an Aurora Scientific Inc model 1300A. Mice were placed supine on a thermostatically controlled table under inhaled anesthesia (4\% to $5 \%$ isoflurane for induction in an induction chamber for less than a minute, and then $\sim 2 \%$ isoflurane via a nosecone for maintenance, both with 1 to $1.5 \mathrm{~L} / \mathrm{min} 100 \% \mathrm{O}_{2}$ ). The knee was kept stationary and the foot was firmly fixed to a footplate, which was connected to the shaft of the motor. The foot was aligned at $90^{\circ}$ to the tibia. Muscle contraction (plantar flexion) was elicited by placing subcutaneous electrodes to stimulate the sciatic nerve. A force frequency protocol was performed by applying a series of stimulation frequencies from 25 to $250 \mathrm{~Hz}$ at $25-\mathrm{Hz}$ increments with a pause of 1 minute between stimuli, and maximal isometric torque was measured at full tetanic contraction at each frequency. 
Histology. Hearts were fixed in 2.5\% glutaraldehyde and $1 \%$ paraformaldehyde in $0.12 \mathrm{M}$ sodium cacodylate buffer, $\mathrm{pH} 7.4$, after which they were embedded in paraffin and sectioned. The sections were stained with hematoxylin and eosin (H\&E) using standard methods and imaged on a NanoZoomer 2.0 (Hamamatsu).

Transthoracic echocardiography. Cardiac ultrasound was performed with a high-frequency linear array ultrasound system (Vevo 2100, VisualSonics) and MS-400 $30 \mathrm{MHz}$ transducer (VisualSonics). Standardized parasternal short-axis M-mode images of the left ventricle were obtained at the level of midpapillary muscles by making a $90^{\circ}$ clockwise rotation of the imaging probe from the parasternal long-axis view. Left ventricle systolic and diastolic posterior and anterior wall thicknesses and end-systolic and end-diastolic internal left ventricle chamber dimensions were measured using the leading-edge method. Left ventricle functional values of fractional shortening and ejection fraction $(\% \mathrm{EF})$ were calculated from the wall thicknesses and chamber dimension measurements using system software. Mice 3 to 4.5 months of age were lightly anesthetized with isoflurane at $5 \%$ for less than 1 minute and then maintained at $1.75 \%$ via nosecone during imaging and were placed on a heated platform equipped with ECG and respiratory rate monitors. For acute response to adrenergic stimulation, data were collected at baseline and 1, 2, 3, and 4 minutes following intraperitoneal injection of $2 \mathrm{mg} / \mathrm{kg}$ isoproterenol.

Cardiac $I / R$. The cardiac I/R protocol was performed as previously described $(12,13)$. Briefly, hearts were quickly dissected into ice-cold Krebs buffer, cannulated via the aorta, and perfused through a Langendorff apparatus in a retrograde fashion with Krebs buffer at a constant pressure of $100 \mathrm{~cm}$ of water at $37^{\circ} \mathrm{C}$. Hemodynamic parameters were measured via a water-filled latex balloon inserted into the left ventricle. Hearts were subjected to 20 minutes of equilibration, 20 minutes of no-flow global ischemia, followed by 90 minutes of reperfusion. Recovery of left ventricular developed pressure (LVDP), rate pressure product (RPP) were measured, and at the end of the reperfusion period infarct size was analyzed via $1 \%(\mathrm{w} / \mathrm{v})$ 2,3,5-triphenyltetrazolium chloride (TTC) staining. Where indicated, CsA or vehicle (DMSO) was added in the perfusate at a concentration of $0.2 \mu \mathrm{M}$ for 5 minutes before ischemia.

Muscle fiber isolation and calcium imaging. The FDB muscle was dissected from the bottom of the mouse foot and digested in collagenase in an agitated water bath at $37^{\circ} \mathrm{C}$ for 1 hour (49). After digestion, individual fibers were released by gentle trituration in Tyrode's buffer with $2 \mu \mathrm{M}$ Rhod-2 AM (Thermo Fisher Scientific) and plated on Matrigel-coated dishes. After 1 hour incubation with Rhod- 2 at $37^{\circ} \mathrm{C}$, the buffer was gently exchanged with Tyrode's buffer containing 100 nM MitoTracker Green (Thermo Fisher Scientific) without Rhod-2 to wash out the Rhod-2. Fibers were imaged 20 minutes after washing. Rhod-2 and MitoTracker Green fluorescence were simultaneously measured on a Leica upright SP8 microscope using confocal sequential line scanning and internal HyD detectors. Rhod-2 and MitoTracker Green were imaged using $552 \mathrm{~nm}$ and $488 \mathrm{~nm}$ excitation with detection from 590 to $650 \mathrm{~nm}$ and 500 to $550 \mathrm{~nm}$, respectively. Whole-volume images were collected from each fiber using $Z$-stacks. Mitochondrial calcium was quantified relative to the mitochondrial content (Rhod-2/MitoTracker Green) per pixel. Images were analyzed in Fiji ImageJ software by thresholding on MitoTracker Green signal to create a mitochondrial mask, and mitochondrial calcium to content ratio was calculated by normalizing the Rhod-2 fluorescence by the MitoTracker Green fluorescence in the pixels contained in the mitochondrial mask. This analysis was performed for the entire 3D volume of each fiber.

For mitochondrial calcium uptake upon caffeine stimulation, instead of $Z$-stacks, time series measurements of Rhod-2 and MitoTracker Green were taken every 2 seconds on a Zeiss 880 confocal microscope. At 20 seconds, $20 \mathrm{mM}$ caffeine was added, and images were obtained up to 5 minutes. As above, images were analyzed in Fiji ImageJ software by thresholding on MitoTracker Green signal to create a mitochondrial mask. The Rhod-2 signal for each fiber was normalized to baseline and the peak mitochondrial calcium fold-change after caffeine was computed in MatLab.

Statistics. Data are represented as mean \pm standard deviation (SD). All data were analyzed for statistical significance using GraphPad Prism software. All 2-way comparisons used unpaired $t$ test with Welch's correction with 2 -tailed $P$ value. A $P$ value less than 0.05 was considered significant. When more than 2 comparisons were made, data were first analyzed by 1-way ANOVA, followed by unpaired 2-tailed $t$ test with Welch's correction to determine significance for each pair of data sets. Where specified (Figure 4, C and D), data were first analyzed by 2-way ANOVA, followed by Bonferroni's multiple-comparisons test to determine significance for each pair of data sets.

Study approval. All animal studies were done in accordance with the NIH Guide for the Care and Use of Laboratory Animals and with the approval of the NHLBI Animal Care and Use Committee, under protocol H-0177R3. 


\section{Author contributions}

JCL, TF, and EM designed research studies. JCL, NCS, NSG, TBW, RJP, JS, MMF, JL, KMH, SM, and DAS conducted experiments and acquired data. JCL, NCS, NSG, TBW, RJP, JS, and DAS analyzed data. BG assisted with experimental design. CL generated the $E M R E^{-/-}$mouse strain. JCL and EM wrote the manuscript with TF's input.

\section{Acknowledgments}

We are grateful to Michele Allen, Audrey Noguchi, and Morteza Peiravi in the NHLBI Murine Phenotyping Core for help with mouse phenotyping, and Zu-Xi Yu in the NHLBI Pathology Core for assistance with histology. We thank Albert Sek, Helene Rosenberg, and Lee Weinstein for generously providing mice, as well as Baylor, Sanger, Harwell, the EUCOMM consortium, and the MMRRC for the EMRE conditional knockout mice. We are grateful to the members of the Finkel and Murphy labs for thoughtful discussions and feedback. This work was supported by NIH intramural funds, a Leducq Transatlantic Network grant (to EM), and NIH grants 1R01HL142589 (to TF), 1FI2GM117605 (to JCL), and 1K22HL137901 (to JCL).

Address correspondence to: Elizabeth Murphy, 10 Center Drive, Bethesda, Maryland 20814, USA. Phone: 301.496.5828; Email: murphy1@nhlbi.nih.gov. Or to: Toren Finkel, 100 Technology Drive, Pittsburgh, Pennsylvania 15219, USA. Phone: 412.383.4409; Email: finkelt@pitt.edu.

1. Denton RM, McCormack JG. The role of calcium in the regulation of mitochondrial metabolism. Biochem Soc Trans. 1980;8(3):266-268.

2. Glancy B, Willis WT, Chess DJ, Balaban RS. Effect of calcium on the oxidative phosphorylation cascade in skeletal muscle mitochondria. Biochemistry. 2013;52(16):2793-2809.

3. Territo PR, Mootha VK, French SA, Balaban RS. Ca(2+) activation of heart mitochondrial oxidative phosphorylation: role of the F(0)/F(1)-ATPase. Am J Physiol, Cell Physiol. 2000;278(2):C423-C435.

4. Balaban RS. The role of $\mathrm{Ca}(2+)$ signaling in the coordination of mitochondrial ATP production with cardiac work. Biochim Biophys Acta. 2009;1787(11):1334-1341.

5. Kohlhaas M, et al. Elevated cytosolic $\mathrm{Na}^{+}$increases mitochondrial formation of reactive oxygen species in failing cardiac myocytes. Circulation. 2010;121(14):1606-1613.

6. Rasola A, Bernardi P. Mitochondrial permeability transition in $\mathrm{Ca}(2+)$-dependent apoptosis and necrosis. Cell Calcium. 2011;50(3):222-233.

7. Ong SB, Samangouei P, Kalkhoran SB, Hausenloy DJ. The mitochondrial permeability transition pore and its role in myocardial ischemia reperfusion injury. J Mol Cell Cardiol. 2015;78:23-34.

8. Kirichok Y, Krapivinsky G, Clapham DE. The mitochondrial calcium uniporter is a highly selective ion channel. Nature. 2004;427(6972):360-364.

9. Baughman JM, et al. Integrative genomics identifies MCU as an essential component of the mitochondrial calcium uniporter. Nature. 2011;476(7360):341-345.

10. De Stefani D, Raffaello A, Teardo E, Szabò I, Rizzuto R. A forty-kilodalton protein of the inner membrane is the mitochondrial calcium uniporter. Nature. 2011;476(7360):336-340.

11. Holmström KM, et al. Assessment of cardiac function in mice lacking the mitochondrial calcium uniporter. J Mol Cell Cardiol. 2015;85:178-182.

12. Pan X, et al. The physiological role of mitochondrial calcium revealed by mice lacking the mitochondrial calcium uniporter. Nat Cell Biol. 2013;15(12):1464-1472.

13. Parks RJ, et al. Cyclophilin D-mediated regulation of the permeability transition pore is altered in mice lacking the mitochondrial calcium uniporter. Cardiovasc Res. 2019;115(2):385-394.

14. Rasmussen TP, et al. Inhibition of MCU forces extramitochondrial adaptations governing physiological and pathological stress responses in heart. Proc Natl Acad Sci USA. 2015;112(29):9129-9134.

15. Wu Y, et al. The mitochondrial uniporter controls fight or flight heart rate increases. Nat Commun. 2015;6:6081.

16. Luongo TS, et al. The mitochondrial calcium uniporter matches energetic supply with cardiac workload during stress and modulates permeability transition. Cell Rep. 2015;12(1):23-34.

17. Kwong JQ, et al. The mitochondrial calcium uniporter underlies metabolic fuel preference in skeletal muscle. JCI Insight. 2018;3(22):121689.

18. Kwong JQ, et al. The mitochondrial calcium uniporter selectively matches metabolic output to acute contractile stress in the heart. Cell Rep. 2015;12(1):15-22.

19. Wang Y, et al. Structural mechanism of EMRE-dependent gating of the human mitochondrial calcium uniporter. Cell. 2019;177(5):1252-1261.e13.

20. Perocchi F, et al. MICU1 encodes a mitochondrial EF hand protein required for Ca(2+) uptake. Nature. 2010;467(7313):291-296.

21. Plovanich M, et al. MICU2, a paralog of MICU1, resides within the mitochondrial uniporter complex to regulate calcium handling. PLoS ONE. 2013;8(2):e55785.

22. Sancak Y, et al. EMRE is an essential component of the mitochondrial calcium uniporter complex. Science. 2013;342(6164):1379-1382.

23. Tsai MF, et al. Dual functions of a small regulatory subunit in the mitochondrial calcium uniporter complex. Elife. 
2016;5:e15545.

24. Vais $\mathrm{H}$, et al. EMRE is a matrix $\mathrm{Ca}(2+)$ sensor that governs gatekeeping of the mitochondrial $\mathrm{Ca}(2+)$ uniporter. Cell Rep. 2016;14(3):403-410

25. Yamamoto T, et al. Analysis of the structure and function of EMRE in a yeast expression system. Biochim Biophys Acta. 2016;1857(6):831-839.

26. Kovács-Bogdán E, et al. Reconstitution of the mitochondrial calcium uniporter in yeast. Proc Natl Acad Sci USA. 2014;111(24):8985-8990.

27. Liu JC, et al. MICU1 serves as a molecular gatekeeper to prevent in vivo mitochondrial calcium overload. Cell Rep. 2016;16(6):1561-1573.

28. Csordás G, et al. MICU1 controls both the threshold and cooperative activation of the mitochondrial $\mathrm{Ca}^{2+}$ uniporter. Cell Metab. 2013;17(6):976-987.

29. Kamer KJ, Mootha VK. MICU1 and MICU2 play nonredundant roles in the regulation of the mitochondrial calcium uniporter EMBO Rep. 2014;15(3):299-307.

30. Logan CV, et al. Loss-of-function mutations in MICU1 cause a brain and muscle disorder linked to primary alterations in mitochondrial calcium signaling. Nat Genet. 2014;46(2):188-193.

31. Murphy E, Steenbergen C. Mechanisms underlying acute protection from cardiac ischemia-reperfusion injury. Physiol Rev. 2008;88(2):581-609.

32. Lemasters JJ, Theruvath TP, Zhong Z, Nieminen AL. Mitochondrial calcium and the permeability transition in cell death. Biochim Biophys Acta. 2009;1787(11):1395-1401.

33. Vallejo-Illarramendi A, Toral-Ojeda I, Aldanondo G, López de Munain A. Dysregulation of calcium homeostasis in muscular dystrophies. Expert Rev Mol Med. 2014;16:e16.

34. Santulli G, Xie W, Reiken SR, Marks AR. Mitochondrial calcium overload is a key determinant in heart failure. Proc Natl Acad Sci USA. 2015;112(36):11389-11394.

35. Abeti R, Abramov AY. Mitochondrial Ca(2+) in neurodegenerative disorders. Pharmacol Res. 2015;99:377-381.

36. Kuang W, et al. Merosin-deficient congenital muscular dystrophy. Partial genetic correction in two mouse models. J Clin Invest. 1998;102(4):844-852.

37. Skarnes WC, et al. A conditional knockout resource for the genome-wide study of mouse gene function. Nature. 2011;474(7351):337-342.

38. Brüning JC, et al. A muscle-specific insulin receptor knockout exhibits features of the metabolic syndrome of NIDDM without altering glucose tolerance. Mol Cell. 1998;2(5):559-569.

39. Millay DP, et al. Genetic and pharmacologic inhibition of mitochondrial-dependent necrosis attenuates muscular dystrophy. Nat Med. 2008;14(4):442-447.

40. Tufi R, et al. Comprehensive genetic characterization of mitochondrial $\mathrm{Ca}^{2+}$ uniporter components reveals their different physiological requirements in vivo. Cell Rep. 2019;27(5):1541-1550.e5.

41. Bertossi A, et al. Loss of Roquin induces early death and immune deregulation but not autoimmunity. J Exp Med. 2011;208(9):1749-1756.

42. Shull MM, et al. Targeted disruption of the mouse transforming growth factor-beta 1 gene results in multifocal inflammatory disease. Nature. 1992;359(6397):693-699.

43. Yu S et al. Variable and tissue-specific hormone resistance in heterotrimeric Gs protein alpha-subunit (Gsalpha) knockout mice is due to tissue-specific imprinting of the gsalpha gene. Proc Natl Acad Sci U S A. 1998;95(15):8715-8720.

44. Murphy E, Pan X, Nguyen T, Liu J, Holmström KM, Finkel T. Unresolved questions from the analysis of mice lacking MCU expression. Biochem Biophys Res Commun. 2014;449(4):384-385.

45. Zhao H, et al. AMPK-mediated activation of MCU stimulates mitochondrial $\mathrm{Ca}^{2+}$ entry to promote mitotic progression. Nat Cell Biol. 2019;21(4):476-486.

46. Koval OM, et al. Loss of MCU prevents mitochondrial fusion in $\mathrm{G}_{1}-\mathrm{S}$ phase and blocks cell cycle progression and proliferation. Sci Signal. 2019;12(579):eaav1439.

47. Burr AR, Molkentin JD. Genetic evidence in the mouse solidifies the calcium hypothesis of myofiber death in muscular dystrophy. Cell Death Differ. 2015;22(9):1402-1412.

48. Raymond CS, Soriano P. High-efficiency FLP and PhiC31 site-specific recombination in mammalian cells. PLoS ONE. 2007;2(1):e162.

49. Park KH, et al. Assessment of calcium sparks in intact skeletal muscle fibers. J Vis Exp. 2014;(84):e50898. 